\title{
Carrier phase recovery in optical fiber communication systems using high- order modulation formats
}

Zhang, Yunfan, Xu, Tianhua, Ding, Jiazheng, Wang, Ziyihui, Xu, Tongyang, et al.

Yunfan Zhang, Tianhua Xu, Jiazheng Ding, Ziyihui Wang, Tongyang Xu, Jian Zhao, Tiegen Liu, "Carrier phase recovery in optical fiber communication systems using high-order modulation formats," Proc. SPIE 11891, Semiconductor Lasers and Applications XI, 1189103 (9 October 2021); doi: $10.1117 / 12.2601072$

SPIE. Event: SPIE/COS Photonics Asia, 2021, Nantong, Jiangsu, China 


\title{
Carrier Phase Recovery in Optical Fiber Communication Systems Using High-Order Modulation Formats
}

\author{
Yunfan Zhang ${ }^{\mathrm{a}}$, Tianhua Xu $\mathrm{Xu}^{\mathrm{a}, \mathrm{b}, \mathrm{c}}$, Jiazheng Ding ${ }^{\mathrm{a}}$, Ziyihui Wang ${ }^{\mathrm{a}}$, Tongyang $\mathrm{Xu}{ }^{\mathrm{c}}$, Jian Zhao ${ }^{\mathrm{a}}$, \\ and Tiegen $\mathrm{Liu}^{\mathrm{a}}$ \\ a School of Precision Instruments and Opto-Electronics Engineering, Tianjin University, \\ Tianjin 300072, China \\ ${ }^{b}$ University of Warwick, Coventry, CV4 7AL, United Kingdom \\ ${ }^{\mathrm{c}}$ University College London, London, WC1E 6BT, United Kingdom
}

\begin{abstract}
Nowadays, the coherent optical communication system plays an important role in communication field because of large capability and bandwidth. A coherent optical communication, based on high-order modulation and digital signal processing technologies, consists of optical transmitters, optical fiber lines, optical amplifiers and optical receivers. In the high-speed coherent optical communication system, the phase noise from the transmitter laser and the local oscillator laser can significantly degrade the performance of the signal transmission and detection, especially for the systems using high-order modulation format, such as m-ary phase shift keying (mPSK) and m-ary quadrature amplitude modulation (m-QAM). Therefore, investigations on laser phase noise compensation algorithm based on digital signal processing technologies has become more and more significant. In this work, a multi-ring carrier phase recovery algorithm is developed for compensating the laser phase noise in optical fiber communication systems using high-order modulation formats. Degradations on the performance of communication systems due to the laser phase noise have been investigated. The system performance using the proposed algorithm and the conventional Viterbi-Viterbi algorithm were also evaluated in 9-channel and 15channel, 32-Gbaud, Nyquist-spaced QPSK, 16-QAM, 64-QAM and 256-QAM coherent transmission systems with considering the impact of the laser phase noise. It is found that the phase noise leads to stricter constraints on the linewidths of transmitter-side and receiver-side lasers, and it can greatly degrade the achievable information rates in communication systems. Besides, compared to the conventional Viterbi-Viterbi algorithm, which is usually applied in the QPSK system, our proposed algorithm can also well mitigate the laser phase noise in 16-QAM, 64-QAM and 256-QAM optical communication systems.
\end{abstract}

Keywords: Coherent optical communication system, Laser phase noise, Carrier phase recovery, Viterbi-Viterbi algorithm, High-order modulation formats

\section{INTRODUCTION}

Nowadays in the information age, the importance of communication technology has gradually become prominent, and various communication technologies have been developed rapidly. Among optical communication technologies, the coherent optical communication system plays an important role in communication field due to large capacity, long transmission distance and high spectrum efficiency. ${ }^{1,2}$ In recent years, digital signal processing (DSP) has evolved vigorously, and the use of DSP technology at the optical receiver to solve various problems in the transmission of optical signal has gradually become a hot issue in the optical communication field. In fact, the application of DSP technology has significantly improved the performance of the coherent optical system. During the process of optical signal transmission, the phase noise from the laser will increase the bit error rate(BER) and degrade the signal-to-noise ratio(SNR), which is one of the important factors affecting the performance of the coherent optical communication system.

Further author information: (Send correspondence to Tianhua Xu and Jian Zhao.)

Tianhua Xu: E-mail: tianhua.xu@ieee.org

Jian Zhao: E-mail: enzhaojian@tju.edu.cn 
With the development of DSP technology, carrier phase recovery algorithms based on DSP technology have attracted extensive attention. In 2006, Pfau T et al. from the University of Stuttgart in Germany conducted the first real-time coherent optical communication experiment. ${ }^{3}$ They successfully achieved laser phase noise compensation for the $800 \mathrm{Mb} / \mathrm{s}$ quadrature phase shift keying(QPSK) system using DSP technology. In 2010, Fatadin I et al. from the National Physical Laboratory of Teddington United Kingdom proposed a carrier phase recovery algorithm based on the Viterbi-Viterbi algorithm to perform QPSK partitioning on the Square 16-QAM system, which reduced the complexity of the hardware. ${ }^{4}$ In 2015, Syed et al. from Politecnico di Torino proposed a novel low-complexity two-stage digital feedforward carrier phase recovery algorithm. ${ }^{5}$ At the BER of $10^{-2}$, for an OSNR penalty of $1 \mathrm{~dB}$, the scheme in this paper could tolerate a linewidth-symbol duration product $\Delta v T_{s}$ of $3.7 \times 10^{-5}$.

In this work, we have investigated the impact of laser phase noise and have developed a modified ViterbiViterbi (MVV) algorithm to compensate for laser phase noise. Numerical simulations have been carried out in a multi-channel, 32-Gbaud Nyquist-spaced wavelength division multiplexing (WDM) optical communication system using the EDC, where the number of channels is set to 9 and 15 , respectively. It is found that the phase noise leads to strict constraints on the linewidths of transmitter-side and receiver-side lasers, and it can greatly degrade the achievable information rates in communication systems. Besides, simulation results indicate that compared to the conventional Viterbi-Viterbi algorithm, which is usually applied in the QPSK system, the proposed algorithm can also well mitigate the laser phase noise in 16-QAM, 64-QAM and 256-QAM optical communication systems.

\section{LASER PHASE NOISE AND MODIFIED VITERBI-VITERBI ALGORITHM}

\subsection{Principle of Laser Phase Noise}

The noise of the semiconductor laser mainly arises from the spontaneous radiation. Each spontaneously radiated photon will add a part of the random field component to the coherent field, disturbing the amplitude and the phase of the original coherent field. ${ }^{6}$ Since $R_{s p}$ (the rate of spontaneous radiation when entering the lasing mode) of the semiconductor laser is relatively high, this spontaneous radiation can occur frequently, which makes the intensity and the phase of the output signal fluctuate. The intensity fluctuations will degrade the signal-to-noise ratio (SNR) of the semiconductor laser, while phase fluctuations will result in a limited frequency linewidth.

The laser phase noise can be described $\operatorname{as}^{7,8}$

$$
\varphi_{t}=\sum_{i=-\infty}^{t} N_{i}
$$

where $N_{i}$ is an independent and identically distributed random Gaussian variable, of which the variance can be described as

$$
\sigma_{f}^{2}=2 \pi \Delta v / R_{s}=2 \pi\left(\Delta v T_{s}\right)
$$

where $\Delta v$ represents the linewidth, $R_{s}$ denotes the symbol rate, and $T_{s}$ represents the symbol period. As Equation (2) reveals, the laser phase noise variance increases with the linewidth of the laser and the symbol period of the transmission system. Besides, the laser phase noise will affect the performance of optical communication system, and will degrade the SNR and increase the BER.

\subsection{Modified Viterbi-Viterbi Carrier Phase Recovery}

The Viterbi-Viterbi algorithm is also known as the M-th power carrier phase recovery algorithm, which is mainly used to compensate the laser phase noise of the signal in the multi-ary phase shift keying (M-PSK) communication system. ${ }^{9,10}$

In this paper we have improved the traditional Viterbi-Viterbi algorithm: The core idea of the algorithm is still to perform fourth power processing on the symbol points of which modulation phase conforms to the QPSK modulation format, and remove the modulation phase to estimate the laser phase noise at this symbol 
point. But the difference from the traditional Viterbi-Viterbi algorithm lies in that the algorithm proposed in this paper performs the rotation and the filtering operations on the symbol points on the intermediate ring. ${ }^{11-20}$ Thus the points on the intermediate ring can also be used to estimate the laser phase noise after processing. The algorithm proposed in this paper can use almost all symbol points for laser phase noise estimation, and the effect of the laser phase noise compensation is greatly improved. ${ }^{21-25}$ Taking 16QAM as an example, the principles and steps of the MVV algorithm are described as follows:

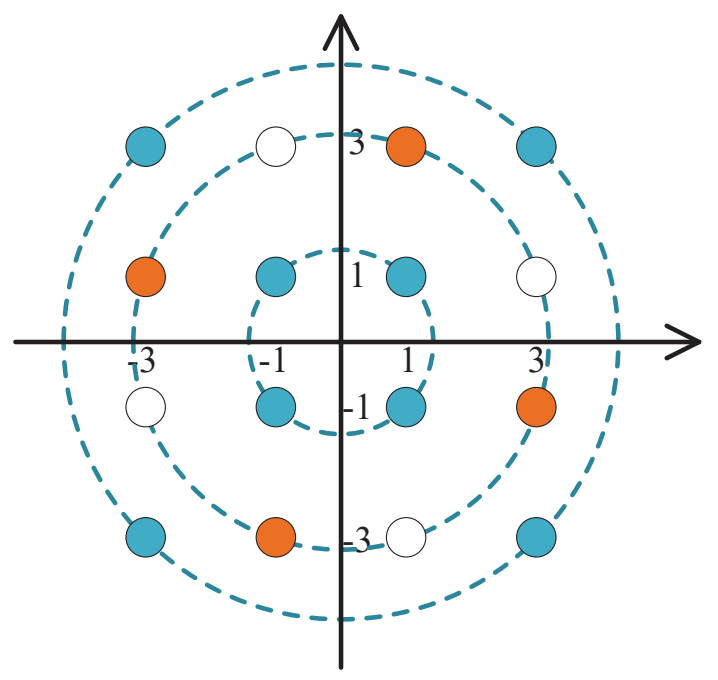

Figure 1. Constellation diagram of 16QAM system

1. Set the threshold and divide the loop

Normalize the input symbol sequence and adjust the amplitude to conform to the coordinate system shown in Figure 1. In this coordinate system, the radii of the three rings of the ideal constellation diagram are $\sqrt{2}, \sqrt{10}$, and $3 \sqrt{2}$ respectively. The first step is to classify all the symbols in the symbol sequence into the innermost ring, middle ring or outermost ring by judging the magnitude of each symbol. In the coordinate system shown in Figure 1, the two thresholds of the ring division can be set as follows:

$$
\begin{gathered}
T h r_{1}=(\sqrt{2}+\sqrt{10}) / 2 \\
T h r_{2}=(3 \sqrt{2}+\sqrt{10}) / 2
\end{gathered}
$$

Comparing the amplitude of each symbol of the input symbol sequence with $T h r_{1}$ and $T h r_{2}$ in turn, all points in the symbol sequence can be divided into two categories: the points, of which the amplitudes are less than $T h r_{1}$ or larger than $T h r_{2}$, are classified as inner ring and outer ring, denoted as $S_{1}$; the points, of which the amplitudes are between $T h r_{1}$ and $T h r_{2}$ are classified as the points on the middle ring, denoted as $S_{2}$. The modulation phase of $S_{1}$ is one of $\pi / 4,3 \pi / 4,5 \pi / 4$, and $7 \pi / 4$, which means the traditional ViterbiViterbi algorithm can be used directly to remove the modulation phase, while the modulation phases of the symbols in $S_{2}$ are not the same as the modulation phase of the QPSK modulation format.

2. Perform fourth Power processing on $S_{1}$

From the Viterbi-Viterbi algorithm, we can know that the QPSK signal $\exp \left(i\left(k \pi / 4+\varphi_{0}\right)\right)$ can be perform on fourth power processing to obtain $-\exp \left(4 i \varphi_{0}\right)$. In order to compensate the -1 that appears after the fourth power processing, the symbol in $S_{1}$ is multiplied by $\exp (i \pi / 4)$ before being processed to the fourth power, which is denoted as $\left(S_{1}\right)^{4}$. 
3. Rotate the middle ring and perform the fourth power processing on $S_{2}$

Observing the constellation diagram in Figure 1, we can see that the eight symbol points on the middle ring can be divided into two types: red symbol points and white symbol points. The phases of the red symbol points are respectively angle $(1+3 i), \pi / 2+$ angle $(1+3 i), \pi+$ angle $(1+3 i)$ and $3 \pi / 2+$ angle $(1+3 i)$. The phases of the white points are respectively angle $(3+1 i), \pi / 2+$ angle $(3+1 i), \pi+$ angle $(3+1 i)$ and $3 \pi / 2+$ angle $(3+1 i)$. Among them, angle $(1+3 i)$ and angle $(3+1 i)$ represent respectively the angle of $1+3 i$ and the angle of $3+1 i$.

The phases of the red symbol points can be performed on fourth power processing after being transformed into $\pi / 4,3 \pi / 4,5 \pi / 4,7 \pi / 4$ by being rotated clockwise by $($ angle $(1+3 i)-\pi / 4)$. In the same way, the phases of the white symbol points can be performed on fourth power processing after being transformed into $\pi / 4,3 \pi / 4,5 \pi / 4,7 \pi / 4$ by being rotated anticlockwise by (angle $(1+3 i)-\pi / 4)$.

Therefore, multiply $S_{2}$ by $\exp (i(\theta-\pi / 4))$ then perform the fourth power processing, and denote the result as anticlc $\left(S_{2}\right)^{4}$, where $\theta=\operatorname{angle}(1+3 i)$. After rotating the middle ring anticlockwise, the modulation phase of the white symbol points in the figure can be removed by fourth power processing, which are the correct points and should be kept, while the red symbol points rotated anticlockwise are wrong points and need to be eliminated. In the same way, after multiplying $S_{2}$ by $\exp (-i(\theta-\pi / 4))$ to rotate it clockwise and denoting the result as $\operatorname{clc}\left(S_{2}\right)^{4}$, the red symbol points will become the correct point, while the white symbol points will become the wrong point.

4. Filter out the correct points, and estimate the laser phase noise

In general, we need to eliminate the additive white Gaussian noise before estimating the laser phase noise. Adding all the symbols in a continuous symbol block can greatly eliminate additive white Gaussian noise because the amplitude of the additive white Gaussian noise conforms to the Gaussian distribution. Since the laser phase noise changes continuously and slowly, it can be considered that the laser phase noise of each symbol in a symbol sequence within an appropriate length is approximately the same.

Considering the existence of the laser phase noise and the additive white Gaussian noise comprehensively, this paper adopts the following scheme to estimate the laser phase noise:

Set a sliding window with a fixed length, sum all the $\operatorname{anticlc}\left(S_{2}\right)^{4}$ and $\operatorname{clc}\left(S_{2}\right)^{4}$ in the window and normalize them respectively, denoted as $\Sigma\left(\text { anticlc } S_{2}\right)^{4}$ and $\Sigma\left(c l c S_{2}\right)^{4}$. Subsequently, take the points of the innermost and outermost rings as the reference standard and filter out the correct symbols from $\Sigma$ anticlc $\left(S_{2}\right)^{4}$ and $\Sigma c l c\left(S_{2}\right)^{4}$.

After normalizing the symbols belonging to $\operatorname{\Sigma anticlc}\left(S_{2}\right)^{4}$ and $\Sigma c l c\left(S_{2}\right)^{4}$ in the window, subtract them from $\Sigma\left(S_{1}\right)^{4}$ respectively, take the amplitude, and denote it as abs. If abs is less than the threshold Thr, then abs is considered to be small enough and the symbol is the correct symbol. After selecting all the correct points in the window in this way, sum all the correct points to eliminate the additive white Gaussian noise and divide the phase of the sum by 4 to obtain the estimated value of the laser phase noise $\varphi_{0}$. Take $\varphi_{0}$ as the estimated value of laser phase noise of the center symbol point of the window. By traversing the sliding symbol block, the laser phase noise of all points in the symbol sequence can be estimated.

\section{Phase unwrapping}

In Matlab, the output range of the angle function is $[-\pi, \pi]$, so the range of the laser phase noise is $[-\pi / 4, \pi / 4]$. When the actual laser phase noise exceeds this range, the step change will bring errors. In order to avoid this situation, it is necessary to unwrap the estimated value of the laser phase noise.

$$
\theta_{n}= \begin{cases}\theta_{n}-\pi / 2, & \left.\theta_{n}-\theta_{(} n-1\right) \geq \pi / 4 \\ \theta_{n}, & \text { pi/4> } \left.\theta_{n}-\theta_{(} n-1\right)>-\pi / 4 \\ \theta_{n}+\pi / 2, & \left.\theta_{n}-\theta_{(} n-1\right) \leq-\pi / 4\end{cases}
$$

Compare $\theta_{n}$ estimated in the current window with $\theta_{n-1}$ estimated in the previous window. If $\theta_{n}$ is too large, decrease it by $\pi / 2$; if $\theta_{n}$ is too small, increase it by $\pi / 2$. 
6. Perform phase noise compensation on the symbol sequence

Finally, the signal is restored with the estimated phase fluctuation to complete the carrier phase recovery.

As for DP-64QAM system and DP-256QAM system, from Figure 2 and Figure 3 we could see that the core idea of the algorithm is the same as DP-16QAM system and in this paper we will not illustrate specific steps.

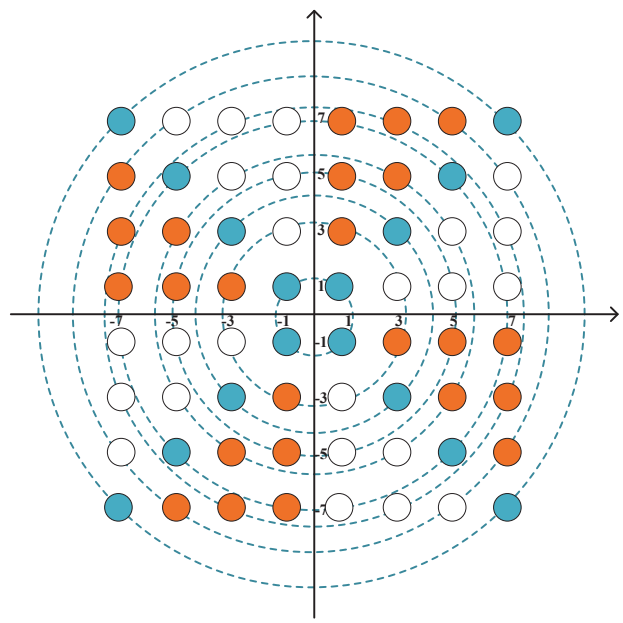

Figure 2. Constellation diagram of 64QAM system

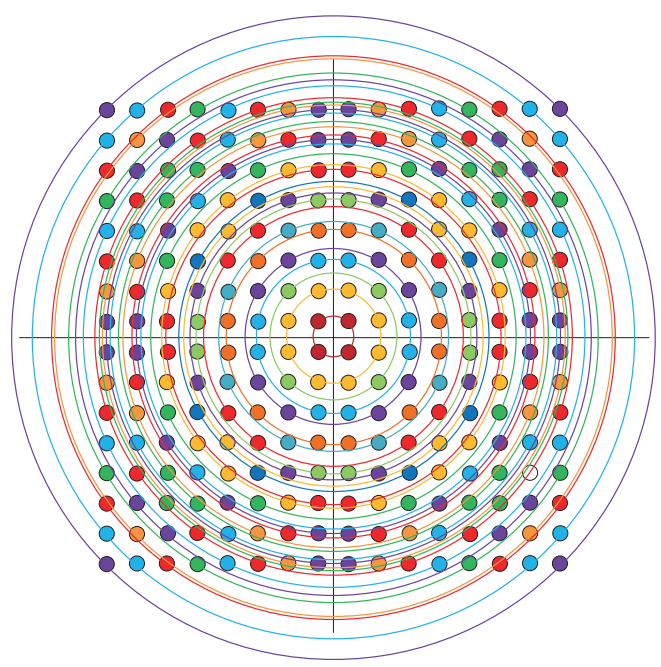

Figure 3. Constellation diagram of 256QAM system

\section{TRANSMISSION SETUP}

Figure 4 illustrates the transmission system setup of the multi-channel 32-Gbaud Nyquist-spaced wavelength division multiplexing (WDM) optical communication system using the following modulation formats: DP-16QAM, DP-64-QAM, and DP-256-QAM. Detailed parameters of the transmission system are shown in Table 1. 
At the transmitter, a $32-\mathrm{GHz}$ spaced laser comb with a center wavelength of $1550 \mathrm{~nm}$ serves as the optical carrier. Subsequently, the WDM optical carriers are modulated respectively by 32-Gbaud signals via in-phase and quadrature (I-Q) modulators. Besides, the transmitted symbol sequences in each channel are random and independent. Based on the split-step Fourier solution of nonlinear Schrödinger equation (NLSE), the SSMF is simulated, and the attenuation coefficient $(\alpha)$, chromatic dispersion coefficient $(D)$, nonlinear coefficient $(\gamma)$ are shown in Table 1.

At the receiver, to realize the coherent detection, the signals are split into two orthogonal polarizations by the polarization beam splitter (PBS) before they are mixed with the LO laser carrier.

Subsequently, the signals are sampled by the analog-to-digital converters (ADCs). After the compensation of chromatic dispersion using DSP module, ${ }^{26}$ the CPR module is used to compensate for the laser phase noise generated from the lasers at the transmitter and the receiver. The frequency offset and the PMD are neglected in all simulations.

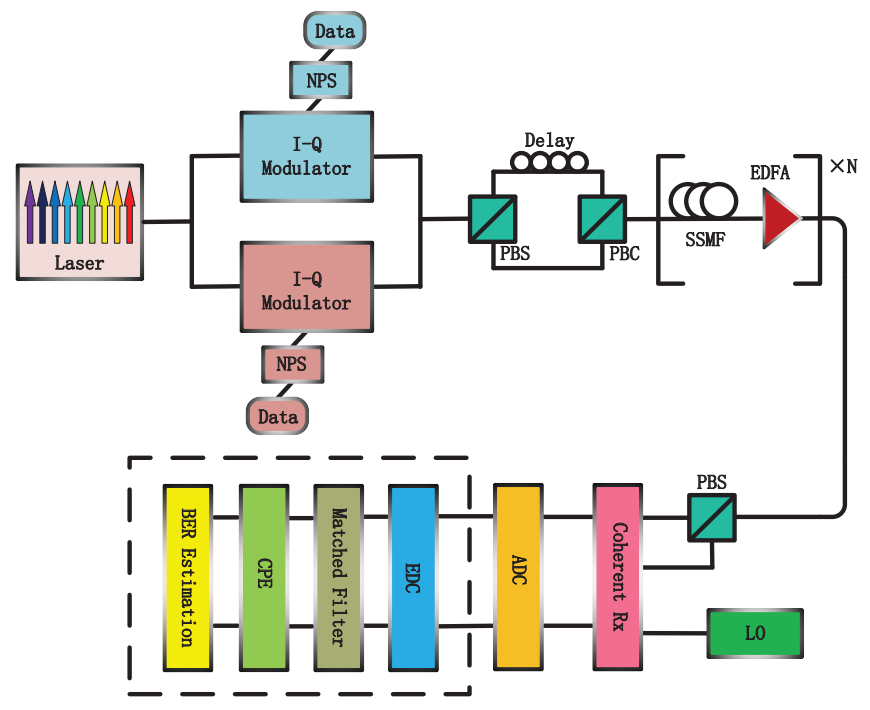

Figure 4. Schematic of the 9-channel Nyquist-spaced optical fibre communication system using EDC. (NPS: Nyquist pulse shaping, PBS: polarisation beam splitter, PBC: polarisation beam combiner, LO: local oscillator, ADC: analogue-to-digital convertor)

Table 1. Transmission System Parameters

\begin{tabular}{|l|l|}
\hline Parameter & Value \\
\hline Symbol rate & $32 \mathrm{Gbaud}$ \\
\hline Channel Spacing & $32 \mathrm{GHz}$ \\
\hline Central wavelength(both transmitter and LO) & $1550 \mathrm{~nm}$ \\
\hline Number of Channels & 9 and 15 \\
\hline Roll-off & $0.1 \%$ \\
\hline Attenuation coefficient $(\alpha)$ & $0.2 \mathrm{~dB} / \mathrm{km}$ \\
\hline Chromatic dispersion $\operatorname{coefficient}(D)$ & $17 \mathrm{ps} / \mathrm{nm} / \mathrm{km}$ \\
\hline Nonlinear coefficient $(\gamma)$ & $0 / \mathrm{W} / \mathrm{km}$ \\
\hline Span length & $80 \mathrm{~km}$ \\
\hline EDFA noise figure & $544 \mathrm{GHz}$ \\
\hline
\end{tabular}




\section{RESULTS AND DISCUSSIONS}

Numerical simulations have been carried out in the 32-Gbaud DP-16QAM transmission system with different scenarios. In all simulations we keep the linewidth of the Tx laser the same as that of the LO laser.

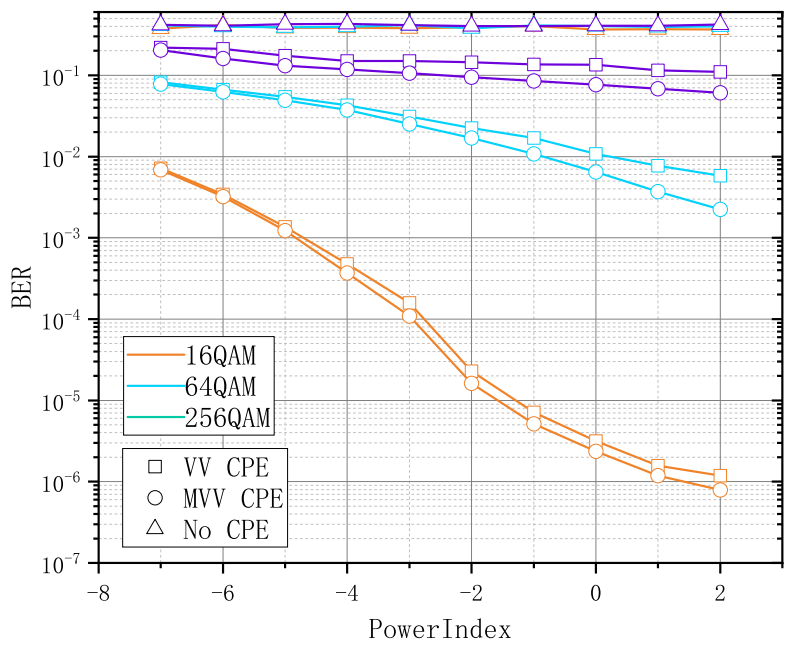

Figure 5. BER versus optical launch power in the 9-ch, 32-Gbaud back-to-back transmission system with the linewidth of $1.5 \mathrm{MHz}$ under DP-16QAM, DP-64QAM and DP-256QAM modulation formats.

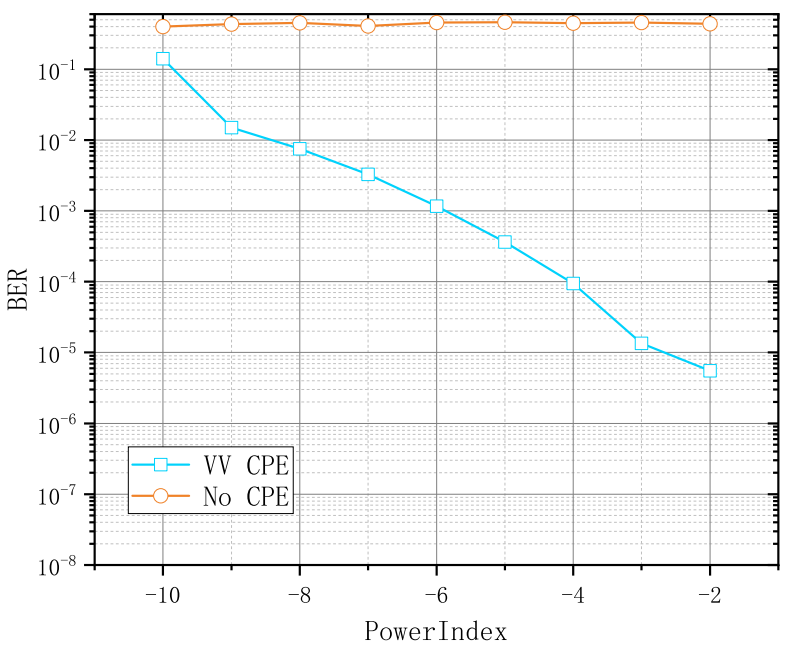

Figure 6. Simulated curves of BER versus optical launch power in the 9-ch 32-Gbaud DP-QPSK transmission system with the linewidth of $1.5 \mathrm{MHz}$ over the $40 \times 80 \mathrm{~km}$ field link.

First we carried out an simulation in the 9-ch 32-Gbaud back-to-back transmission system with the linewidth of $1.5 \mathrm{MHz}$. Figure 5 provides the variation of BER versus optical launch power under different modulation formats, processed respectively by Viterbi-Viterbi carrier phase recovery algorithm(VV CPR) and Modified 
Viterbi-Viterbi carrier phase recovery algorithm(MVV CPR). From the figure we can see that both VV CPR and MVV CPR greatly suppress the laser phase noise, and the effects of MVV CPR behaves better than VV $\mathrm{CPR}$ in all modulation formats.

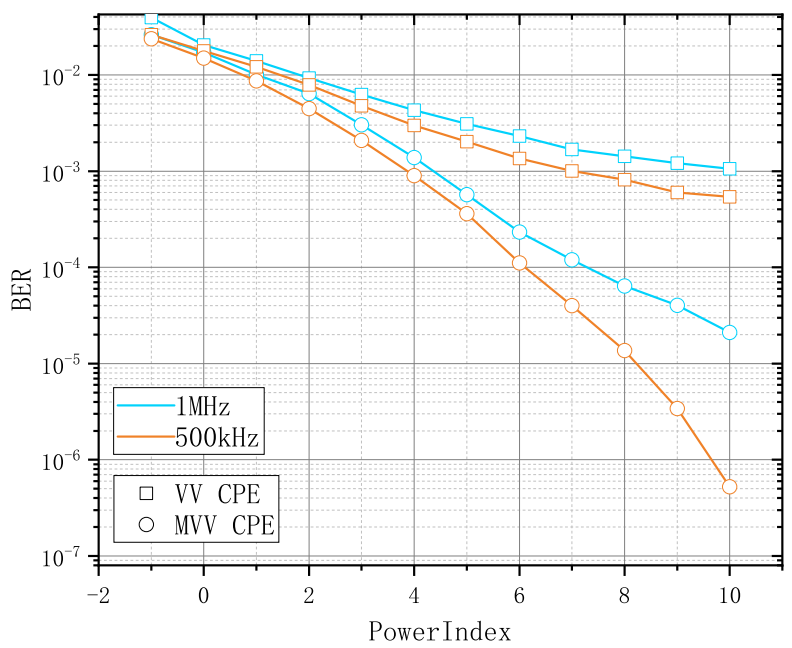

Figure 7. The variation of BER versus optical launch power of $500 \mathrm{kHz}$ and $1 \mathrm{MHz}$ processed respectively by VV CPR and MVV CPR in the 9ch DP-64QAM system.

Then simulations have been implemented in the 9-ch 32-Gbaud DP-QPSK transmission system with the linewidth of $1.5 \mathrm{MHz}$ over the $40 \times 80 \mathrm{~km}$ field link. From Figure 6 we can see that Viterbi-Viterbi carrier phase recovery algorithm could significantly compensate the laser phase noise.

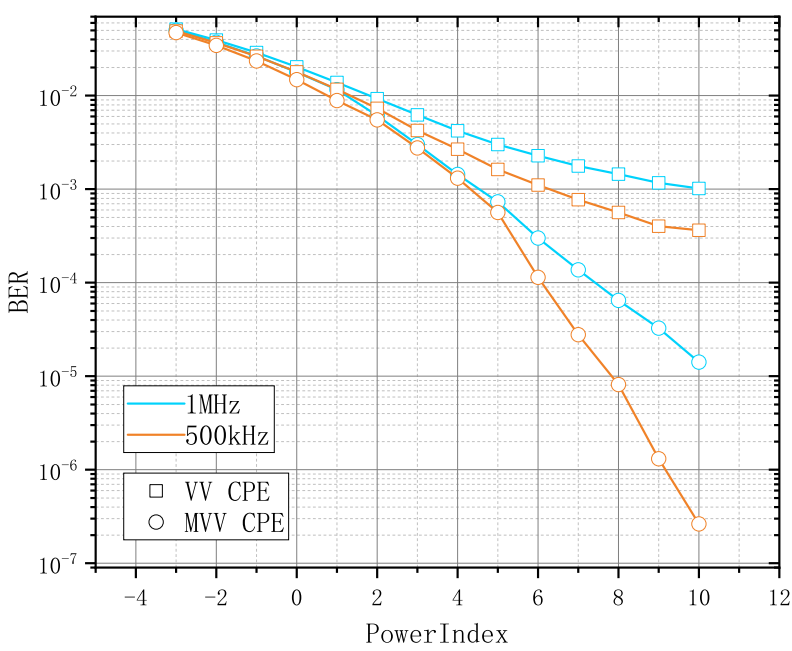

Figure 8. The variation of BER versus optical launch power of $500 \mathrm{kHz}$ and $1 \mathrm{MHz}$ processed respectively by VV CPR and MVV CPR in the 15-ch DP-64QAM system. 
Further simulations were also performed in the 9-ch 32-Gbaud DP-64QAM transmission system and 15-ch 32-Gbaud DP-64QAM transmission system over the $10 \times 80 \mathrm{~km}$ field link with different linewidths. The variation of BER versus optical launch power of $500 \mathrm{kHz}$ and $1 \mathrm{MHz}$ processed respectively by VV CPR and MVV CPR in the 9ch system are summarized in Figure 7, and the results of $15 \mathrm{ch}$ system are illustrated in Figure 8. Compared with the results of the back-to-back system, the advantages of MVV are more prominent here. In the same system, the BER of the data processed by MVV CPR behaves one order of magnitude lower than that of the data processed by VV CPR. This gap can be further increased as the optical launch power grows. As shown in Figure 7 and Figure 8, the maximum improvement reaches three orders of magnitude. Besides, it can be seen that the reduction in BER caused by MVV can completely offset the damage to system performance caused by the $500 \mathrm{kHz}$ increase in linewidth (from $500 \mathrm{kHz}$ to $1 \mathrm{MHz}$ ). When the optical launch power is large enough, the BER of the system with the linewidth of $1 \mathrm{MHz}$ processed by MVV CPR can be even one order of magnitude lower than that of the system with the linewidth of $500 \mathrm{kHz}$ processed by VV CPR.

\section{CONCLUSIONS}

In this work, we have studied the impact of the laser phase noise and have developed a modified Viterbi-Viterbi carrier phase recovery algorithm. Numerical simulations have been carried out to illustrate the improvement in system performance caused by MVV CPR compared to traditional VV CPR. Simulation results indicate that the BER improvement by MVV can reach one order of magnitude under the same linewidth. Besides, the BER reduction brought by MVV can offset the performance degradation from the increase of laser linewidth by 500 $\mathrm{kHz}$. Specifically, when the optical launch power is large enough, the BER of the system with the linewidth of $1 \mathrm{MHz}$ processed by MVV CPR could be one order of magnitude lower than the BER of the system with the linewidth of $500 \mathrm{kHz}$ processed by the traditional VV CPR.

\section{ACKNOWLEDGMENTS}

This work is supported by EU Horizon 2020 Grant 101008280 and Tianjin University Independent Innovation Fund (2021XYK-0004).

\section{REFERENCES}

[1] Agrawal, G. P., [Fiber-optic communication systems], vol. 222, John Wiley \& Sons (2012).

[2] Xu, T., Shevchenko, N. A., Zhang, Y., Jin, C., Zhao, J., and Liu, T., "Information rates in Kerr nonlinearity limited optical fiber communication systems," Opt. Express 29(11), 17428-17439 (2021).

[3] Pfau, T., Hoffmann, S., Peveling, R., Bhandare, S., Ibrahim, S. K., Adamczyk, O., Porrmann, M., Noé, R., and Achiam, Y., "First real-time data recovery for synchronous QPSK transmission with standard DFB lasers," IEEE Photon. Technol. Lett. 18(18), 1907-1909 (2006).

[4] Fatadin, I., Ives, D., and Savory, S. J., "Laser linewidth tolerance for 16-QAM coherent optical systems using qpsk partitioning," IEEE Photon. Technol. Lett. 22(9), 631-633 (2010).

[5] Bilal, S. M., Bosco, G., Cheng, J., Lau, A. P. T., and Lu, C., "Carrier phase estimation through the rotation algorithm for 64-QAM optical systems," J. Light. Technol. 33(9), 1766-1773 (2015).

[6] Yamamoto, Y. and Kimura, T., "Coherent optical fiber transmission systems," IEEE J Quantum Electron 17(6), 919-935 (1981).

[7] Kahn, J. and Kazovsky, L., "Coherent optical communications: Fundamentals and future prospects," in [Frontiers in Optics], FThI1, Optical Society of America (2006).

[8] Kikuchi, K., "Characterization of semiconductor-laser phase noise and estimation of bit-error rate performance with low-speed offline digital coherent receivers," Opt. Express 20(5), 5291-5302 (2012).

[9] Viterbi, A., "Nonlinear estimation of PSK-modulated carrier phase with application to burst digital transmission," IEEE Trans. Inf. Theory 29(4), 543-551 (1983).

[10] Zafra, S. O., Pang, X., Jacobsen, G., Popov, S., and Sergeyev, S., "Phase noise tolerance study in coherent optical circular qam transmissions with viterbi-viterbi carrier phase estimation," Optics express 22(25), 30579-30585 (2014). 
[11] Noé, R., "Phase noise-tolerant synchronous QPSK/BPSK baseband-type intradyne receiver concept with feedforward carrier recovery," J. Light. Technol. 23(2), 802-808 (2005).

[12] Li, Y., Zheng, Q., Xie, Y., Han, J., and Li, W., "Low complexity carrier phase estimation for m-QAM optical communication systems," Photonic Netw. Commun. 38(1), 121-128 (2019).

[13] Navarro, J. R., Kakkar, A., Pang, X., Ozolins, O., Schatz, R., Olmedo, M. I., Jacobsen, G., and Popov, S., "Carrier phase recovery algorithms for coherent optical circular mQAM systems," J. Light. Technol. 34(11), 2717-2723 (2016).

[14] Ip, E. and Kahn, J. M., "Feedforward carrier recovery for coherent optical communications," J. Light. Technol. 25(9), 2675-2692 (2007).

[15] Fang, X. and Zhang, F., "Phase noise estimation and suppression for PDM CO-OFDM/OQAM systems," J. Light. Technol. 35(10), 1837-1846 (2017).

[16] Lippiatt, D., Varughese, S., Richter, T., Tibuleac, S., and Ralph, S. E., "Joint linear and nonlinear noise estimation of optical links by exploiting carrier phase recovery," in [Optical Fiber Communication Conference], Th2A-49, Optical Society of America (2020).

[17] Jin, C., Shevchenko, N. A., Li, Z., Popov, S., Chen, Y., and Xu, T., "Nonlinear coherent optical systems in the presence of equalization enhanced phase noise," J. Light. Technol. , 4646 - 4653 (2021).

[18] Nguyen, T. T., Boscolo, S., Ali, A., Tan, M., Zhang, T., Takasaka, S., Sugizaki, R., Sygletos, S., and Ellis, A. D., "Kernel-based learning-aided phase noise compensation in dual-pump optical phase conjugation coherent system," in [2021 Optical Fiber Communications Conference and Exhibition (OFC)], 1-3, IEEE (2021).

[19] Yan, X., Cao, C., Zhang, W., Zeng, X., Feng, Z., Wu, Z., Su, X., and Wang, T., "Low-complexity carrier phase estimation for M-ary quadrature amplitude modulation optical communication based on dichotomy," Opt. Express 28(17), 25263-25277 (2020).

[20] Deynu, F. K., Akpari, E. W., and Akama, C., "Phase noise effects on the performance of joint carrier phase recovery algorithms in phase-locked WDM superchannel transmission systems," Opt. Fiber Technol. 54, 102104 (2020).

[21] Kim, S.-Y., Suzuki, T., Kani, J.-I., and Yoshida, T., "Carrier phase estimation softwarized on GPU using decision-aided phase unwrapping for flexible optical coherent access systems," J. Light. Technol. 39(6), 1706-1714 (2021).

[22] Li, C., Pan, D., Feng, Y., Lin, J., Xi, L., Tang, X., Zhang, W., and Zhang, X., "Carrier phase estimation scheme for faster-than-nyquist optical coherent communication systems," Chin Opt Lett 14(10), 100601 (2016).

[23] Neves, M. S., Monteiro, P. P., and Guiomar, F. P., "Enhanced phase estimation for long-haul multi-carrier systems using a dual-reference subcarrier approach," J. Light. Technol. 39(9), 2714-2724 (2021).

[24] Zhong, K., Ke, J. H., Gao, Y., and Cartledge, J. C., "Linewidth-tolerant and low-complexity two-stage carrier phase estimation based on modified QPSK partitioning for dual-polarization 16-QAM systems," $J$. Light. Technol. 31(1), 50-57 (2013).

[25] Yang, T., Shi, C., Chen, X., Zhang, M., Ji, Y., Hua, F., and Chen, Y., "Linewidth-tolerant and multiformat carrier phase estimation schemes for coherent optical m-QAM flexible transmission systems," Opt. Express 26(8), 10599-10615 (2018).

[26] Xu, T., Jacobsen, G., Popov, S., Li, J., Vanin, E., Wang, K., Friberg, A. T., and Zhang, Y., "Chromatic dispersion compensation in coherent transmission system using digital filters," Optics express 18(15), 1624316257 (2010). 